\title{
CONSTRUÇÃO DE UM MATERIAL EDUCATIVO PARA A PREVENÇÃO DO CÂNCER DE COLO DO ÚTERO
}

Ana Carolina Peuker

Universidade do Vale do Rio dos Sinos - Unisinos

Natália Britz de Lima

Universidade do Vale do Rio dos Sinos - Unisinos

Karine de Mello Freire

Universidade do Vale do Rio dos Sinos - Unisinos

Caio Marcelo Miolo de Oliveira Universidade do Vale do Rio dos Sinos - Unisinos

Elisa Kern de Castro Universidade do Vale do Rio dos Sinos - Unisinos

\section{Resumo}

O câncer de colo de útero (CCU) é o terceiro tumor mais incidente nas mulheres brasileiras, e as ações de prevenção primária podem reduzir a mortalidade pela doença. Profissionais devem buscar meios para que a comunicação em saúde seja efetiva, utilizando metodologias práticas e científicas. O objetivo deste artigo é descrever o processo de elaboração de um material educativo produzido por profissionais da psicologia, enfermagem e design, direcionado a usuárias da atenção básica. O conteúdo do material foi desenvolvido a partir de uma pesquisa empírica sobre as percepções de mulheres e seus comportamentos frente ao CCU. A construção do material baseou-se nas teorias de autorregulação em saúde e no human-centered design. O produto deste estudo foi a confecção de uma cartilha em formato de flor, com quatro histórias que abarcam os principais conteúdos relacionados aos comportamentos de prevenção do CCU.

Palavras-chave: Educação em Saúde; Promoção da Saúde; Atenção Primária; Percepção da Doença; Câncer.

\section{EDUCATIONAL BOOKLET CONSTRUCTION TO PREVENT CERVICAL CANCER}

\begin{abstract}
Cervical cancer (CC) is the third most frequent tumor among Brazilian women, and primary prevention care can reduce mortality from the disease. Health professionals should seek resources for communication to be effective, using practical and scientific methodologies. The purpose of the paper is to describe the process of developing an educational booklet prepared by professionals of psychology, nursing and design, aimed at primary care users. The booklet content was produced starting from an empirical research on the perceptions of women and their behavior to deal the CC. The booklet construction was based on health self-regulation model and Human-centered design theories. The product of this study was the construction of a booklet flowershaped, with four stories covering the main factors related to the conduct of preventive CC.

Keywords: Health Education; Health Promotion; Primary Care; Illness Perception; Cancer.
\end{abstract}




\title{
CONSTRUCCIÓN DE UN MATERIAL EDUCATIVO PARA LA PREVENCIÓN DEL CÁNCER CERVICAL
}

\begin{abstract}
Resumen
El cáncer cervical (CC) es el tercer tumor más incidente en mujeres brasileñas, y acciones de prevención primaria pueden reducir la mortalidad por la enfermedad. Los profesionales deben buscar formas para que la comunicación en salud sea efectiva, utilizándose de metodologías prácticas y científicas. El objetivo del artículo es describir el proceso de elaboración de un material educativo producido por profesionales de psicología, enfermería y design, para usuarias de la atención básica. El contenido del material fue producido a partir de una investigación empírica sobre las percepciones de mujeres y sus conductas en relación al CC. La construcción del material fue basado en las teorías de autorregulación en salud y human-centred design. El producto fue la confección de una dobladura en forma de flor, con cuatro historias que engloban los principales contenidos relacionados a las conductas de prevención del CC.

Palabras clave: Educación en Salud; Promoción de la Salud; Atención Primaria; Percepción de la Enfermedad; Cáncer.
\end{abstract}

\section{INTRODUÇÃO}

O câncer de colo de útero (CCU) é o segundo tipo de câncer mais comum em todo o mundo, sendo responsável pela morte de mais de 270 mil mulheres todos os anos (Organização Mundial da Saúde, 2013). No Brasil, este é o terceiro tipo de câncer mais incidente na população feminina, sendo estimado 16.350 novos casos para o ano de 2016 (INCA, 2016). É uma neoplasia maligna de evolução lenta, que têm como causa principal a infecção pelo papiloma vírus humano (HPV) transmitido no contato sexual (Mantenese, 2008; Ministério da Saúde, 2013). A principal forma de prevenção é por meio do exame de Papanicolau e recentemente, houve um grande avanço na prevenção do CCU que foi a inserção da vacina contra o HPV no calendário vacinal do Sistema Único de Saúde do Brasil (Ministério da Saúde, 2015).

Apesar da viabilidade da prevenção do CCU e da relativa simplicidade do diagnóstico das lesões precursoras e da doença, é fato que no Brasil ainda existe um número elevado de mulheres que recebem o diagnóstico tardiamente (Tavafian, 2012). A não adesão às condutas preventivas e de autocuidado podem estar relacionadas com o medo da dor, pudor, dificuldade de acesso aos serviços de saúde, não se sentir em risco de ter a doença e barreiras de conhecimento sobre a importância da prevenção (Brito-Silva, Bezerra, Chaves, \& Tanaka, 2013). Para mudar este panorama, é fundamental a busca de informações precisas sobre comportamentos e crenças que podem interferir na saúde da mulher e na percepção de risco de desenvolver a doença (Bish, Ramirez, Burgess, \& Hunter, 2005).

Um conceito chave para compreender as condutas preventivas em relação à saúde é o de percepção de risco. Os mecanismos de autorregulação envolvidos quando a pessoa está em risco de ter uma doença ainda não estão bem estabelecidos. No entanto, o risco percebido, entendido como a percepção do quanto sua vida está vulnerável em relação a uma determinada doença, pode ser 
um fator representacional chave para as reações e comportamentos relacionados à sua saúde (Marteau \& Weinman, 2013). Assim, as reações relacionadas ao risco de ter câncer podem regular a percepção subjetiva da pessoa, que interage com representações individuais que ela já possui sobre as possíveis causas da doença (Shiloh, 2006). Nesse sentido, as informações que a pessoa tem sobre a doença são o primeiro passo para que ela se perceba em risco ou não de ter determinada doença, mas não o único fator. O indivíduo deve elaborar a informação que possui, identificar e atribuir determinado sintoma ao câncer para que inicie o seu processo pessoal de busca de ajuda (Shiloh, Drori, Orr-Urtreger, \& Friedman, 2009.

Com respeito à prevenção de doenças, a autorregulação do comportamento em saúde deve ser anterior ao aparecimento dos sintomas. Quando há sintoma corporal, esse sintoma ativa o processo autorregulatorio do indivíduo e pode fazer com que ele busque ajuda. Entretanto, no caso de doenças assintomáticas em estágio inicial, como o CCU, os processos que envolvem a conscientização e tomada de atitude para a realização de comportamentos que evitem a doença podem ser bem mais complexos. Além disso, podem depender também da história pessoal do indivíduo (Rees, Fru, Cull, \& Sutton, 2004).

As ações preventivas de doenças visam identificar aspectos e focos problemáticos nos quais é possível desenvolver alguma estratégia de atuação (Ministério da Saúde, 2013). A atenção primária ou atenção básica tem como um dos seus focos a prevenção de doenças e a promoção da saúde a fim de melhorar os indicadores de saúde e reduzir as causas previsíveis de morbimortalidade de diferentes doenças (Cruz \& Loureiro, 2008). Com respeito ao $\mathrm{CCU}$, esforços devem ser realizados para motivar o público feminino a pensar sobre questões que ameaçam a sua saúde e a fomentar o autocuidado e prevenção de doenças. Os profissionais deveriam buscar meios para que a comunicação com as mulheres seja efetiva, lançando mão de metodologias práticas que sejam comprovadas cientificamente (Freitas, Waechter, Coutinho, \& Gubert, 2014).

No campo do design, metodologias podem ser utilizadas para aproximar o público do conteúdo impresso. Um exemplo é a metodologia human-centred design (centrada no ser humano), que incorpora métodos da antropologia e do design participativo para ouvir as pessoas que serão afetadas pelo projeto, incluindo-as no processo de resolução de um desafio estratégico. O processo de projeto inclui três etapas: (1) Ouvir: coletar histórias e inspiração das pessoas; (2) Criar: traduzir o conhecimento oportunidades e soluções; (3) Entregar: realizar a solução por meio de protótipos e planos de implementação (IDEO, 2009).

O objetivo deste estudo foi descrever o processo de construção de um material educativo (cartilha) sobre o CCU, visado a sua prevenção, percepção de 
consequências e tratamento. Para a construção do material utilizou-se como base o contexto de mulheres usuárias de unidades Básicas de Saúde (UBS). Uma equipe interdisiciplinar atuou na elaboração do material, incluindo profissionais da área de Psicologia, Enfermagem e Design.

\section{MÉTODO}

Como método serão citados os passos de elaboração do material educativo.

\section{Subsídios para o conteúdo do material educativo}

A construção do material é o produto final de um projeto maior intitulado Saúde da mulher: fatores psicológicos associados à prevenção e condutas de autocuidado frente ao câncer de colo de útero, aprovado pelo Comitê de Ética em Pesquisa da Secretaria de Saúde de Porto Alegre, processo sob $n^{\circ} 001.008580 .12 .0$, registro no CEP $n^{\circ}$ 756. Buscou-se, através do desenvolvimento deste projeto mais amplo conhecer usuárias das UBS, suas percepções e crenças acerca da doença, o que possibilitou a construção posterior do material educativo.

O projeto foi constituído por três estudos: no estudo I, avaliou-se a percepção e o conhecimento sobre o CCU em mulheres saudáveis e mulheres em risco de vir a ter a doença; no estudo II, foi avaliado o conhecimento dos profissionais de saúde sobre o CCU e suas habilidades sociais; e o estudo III, tratou de uma intervenção com as usuárias de uma UBS para incrementar as condutas preventivas de autocuidado frente ao CCU. No que concerne ao desenvolvimento do material, foram explorados os resultados dos estudos I e III.

No estudo I, realizado no período entre setembro de 2012 a março de 2013, foram entrevistadas 189 mulheres, com idade média de 40,56 anos (DP= $11,59)$ usuárias de duas UBS's de Porto Alegre. Algumas características foram levadas em consideração para a construção do material educativo, expostas na Tabela 1. Em relação à escolaridade a maioria tinha ensino médio completo ou incompleto $53,4 \%(\mathrm{~N}=101)$. Em relação ao comportamento sexual, $77,2 \%$ $(\mathrm{N}=146)$ disseram ter uma vida sexual ativa. $\mathrm{O}$ uso do preservativo era baixo na amostra, sendo $42,3 \%(N=80)$ referiram nunca ter usado preservativo no último ano e $50,8 \% \quad(\mathrm{~N}=96)$ nunca usaram nos últimos três meses. Quando questionadas sobre os serviços de saúde que costumavam frequentar a maioria $70,4 \%(\mathrm{~N}=133)$ referiu a UBS. As mulheres da amostra mostraram ter $\mathrm{o}$ costume de realizar o exame de Papanicolau, sendo que $77,5 \% \quad(\mathrm{~N}=146)$ realizaram no último ano e $85,7 \%(\mathrm{~N}=162)$ o fizeram nos últimos três anos. 0 modo de prevenção do $\mathrm{CCU}$ mais citado foi exames preventivos/consultas por $45 \%(N=85)$ das mulheres, seguido de $27 \%(N=51)$ que afirmaram não se 
prevenir e 16,9\% ( $\mathrm{N}=32)$ afirmaram realizar a prevenção com hábitos de vida saudáveis (alimentação, exercícios físicos).

Estes dados também ajudaram na construção do terceiro estudo no qual foi conduzida uma intervenção psicoeducativa em grupo com as usuárias da UBS a fim de fomentar a prevenção do CCU. Foram realizados dois grupos, com quatro encontros semanais cada e duração de uma hora e meia, no mês de setembro de 2013. Todos os encontros foram coordenados por uma psicóloga com experiência clínica e por pelo menos mais uma profissional da área. A intervenção teve como objetivo central aumentar o conhecimento quanto ao CCU e seus fatores de risco, promover e fortalecer condutas de autocuidado (fazer o exame preventivo) e ajustar a percepção da doença e de risco (torná-las mais realistas). Participaram dos encontros dez mulheres, com idade entre 20 e 70 anos $(M=54,60 ; d p=13,52)$ (Corazza, 2014). Os dados discutidos nos encontros também embasaram a construção do material educativo. A amostra foi selecionada por conveniência, de mulheres que tinham a disponibilidade de participar de todos os encontros.

\section{Sistematização do conteúdo}

A elaboração do conteúdo do material educativo teve como base o Modelo teórico de Autorregulação em saúde (Leventhal et al., 1997) e os dados coletados nas etapas anteriores do estudo. As informações sobre o CCU foram obtidas através de revisão da literatura científica nacional e internacional. Foram utilizados também dados do Instituto Nacional do Câncer (INCA) e do Ministério da Saúde. Buscou-se trabalhar com mulheres em diferentes faixas etárias, por isso optou-se que as personagens tivessem 18, 35, 48 e 66 anos. Apesar de as mulheres com 18 anos não serem alvo de campanhas para participação no exame preventivo, estas devem prevenir-se do HPV e de outras doenças transmissíveis, já relacionando o autocuidado com a saúde sexual.

\section{Elaboração do material educativo}

Na primeira etapa ouvir, a intenção dos designers foi desenvolver empatia pelas pessoas que serão afetadas pelo projeto. Neste sentido, é importante selecionar adequadamente as pessoas e os locais de observação para que histórias significativas sejam coletadas. São usadas técnicas de pesquisa qualitativa, para identificar o conhecimento preexistente e aqueles necessários para responder a meta do projeto (IDEO, 2009).

Esta fase ocorreu entre os meses de janeiro e junho de 2014, buscou-se observar as atividades na UBS, entrevistar as pacientes, buscar inspiração em situações análogas à leitura de um material educativo. Nesta etapa o objetivo foi identificar o perfil das usuárias do serviço do SUS, seus hábitos, comportamentos e interação com diferentes suportes de comunicação. Por meio de registros fotográficos, buscou-se mapear o conjunto de informações as quais essas 
mulheres são expostas ao longo do dia, em diferentes espaços (ônibus, placas na rua, centro de saúde, varejo, igreja, entre outros). A partir disso, foram selecionadas estratégias de comunicação disponíveis em sites de referências de peças gráficas.

A etapa criar diz respeito à interpretação dos dados, a identificação de padrões e oportunidades e a criação de soluções. O objetivo é sintetizar os dados de forma a transportar a equipe de projetistas da inspiração para a ideação. As histórias coletadas no campo são sintetizadas por meio de diferentes ferramentas (personagens, mapas de afinidades) de forma a gerar ideias de projeto. Outro método é o de identificação de estruturas na qual as histórias individuais são inseridas em contextos mais abrangentes utilizando ferramentas como mapas, diagramas e matrizes. As ideias de soluções são esboçadas, desenvolvidas e prototipadas rapidamente como uma maneira de criar estímulos para o diálogo (oportunidades). O protótipo permite avaliar se um conceito é útil, usável, desejável, fácil de usar, viável e possível de ser implementado (IDEO, 2009).

Esta etapa ocorreu entre os meses de julho e setembro de 2014. Foram analisados os materiais impressos que as usuárias tinham contato tanto na UBS, quanto em outros locais, como outras redes de saúde, locais públicos e privados, como ônibus, ruas, parques, escolas, farmácias, entre outros. Os materiais impressos que foram analisados eram ligados à saúde ou sobre temas relacionados ao bem público, como por exemplo, campanha do agasalho, proibição de trabalho infantil, segurança no trânsito, etc. (IDEO, 2009).

\section{RESULTADOS}

Analisando os materiais coletados constataram-se outras similaridades como: a utilização de personagens femininas ilustradas, algumas em linguagem lúdica; presença de figuras públicas, como atrizes ou cantoras nacionais; a cor rosa em predominância sobre outras cores; utilização de atributos e signos visuais relacionados a feminilidade, como, flores, esmaltes, coração, silhuetas femininas. Como naqueles materiais gráficos sobre temas gerais, estes relacionados à saúde da mulher também possuem grande quantidade de conteúdo, que é disponibilizado no material de forma pouco atrativa. Os formatos destes materiais também se limitavam a cartazes, cartilhas e folderes.

$\mathrm{Na}$ busca de inspiração em outros contextos puderam-se encontrar formas alternativas de comunicação. Dentre os modelos encontrados estavam bolsas, folhetos em formato sanfona, pôsteres em composição textual e ilustrativas, dobraduras de papel (origami). Uma das estratégias que pareceu interessante, principalmente por gerar curiosidade em descobrir as respostas, permitindo uma maior interação com o público, foi o do origami. Neste modelo há perguntas na parte interna da dobradura e suas respostas encontram-se ao desdobrar as abas. Além disso, a forma de comunicação deste modelo não é tão onerosa, é 
praticamente o mesmo para impressão de cartazes e cartilhas, só envolve a manipulação de realizar a dobradura.

Nas entrevistas, entendeu-se que as mulheres que frequentam a UBS já têm o hábito de realizar a prevenção em relação ao CCU e possuem algum conhecimento sobre a doença. Na fala das entrevistadas, as pessoas só procuram ajuda quando estão doentes ou quando aparecem sintomas. Identificou-se como oportunidade de projeto: "Como poderíamos estimular as mulheres que não estão nos centros de saúde a procurar esses espaços para realizar a prevenção do câncer de colo de útero?" "Como poderíamos passar a informação sobre o câncer de colo de útero em uma linguagem simples e efetiva"?

Por fim, a etapa entregar concerne a realização e a viabilidade das ideias. Nesta fase, a solução deve ser aperfeiçoada por meio de diferentes protótipos, mini-pilotos e pilotos. Além disso, são criados indicadores para avaliar o resultado alcançado e a partir disso evoluir a proposta (IDEO, 2009). Como resultado desta etapa, foram identificadas características necessárias ao material de comunicação: Possuir formato diferente de cartazes e cartilhas e que possua denotação direta do universo feminino; Utilizar linguagem visual simples, de fácil compreensão; Explorar o uso de ilustrações de personagens femininas contemplando os perfis e características estéticas das pacientes; Expor os textos a partir da lógica pergunta/resposta ou desafio/solução; Explorar a curiosidade das pessoas, de forma que o material seja interativo.

A partir destas diretrizes e de uma analogia com o exame preventivo para o câncer de colo de útero, criou-se um primeiro esboço de um conceito: "bonita por fora e por dentro sempre: abra e descubra como". A referência faz analogia à beleza da mulher, que além de cuidar da aparência física, precisa principalmente cuidar da parte interna, ou seja, da saúde do corpo. O símbolo escolhido para ilustrar o universo feminino e trazer o aspecto de descoberta, de olhar por dentro, foi o de uma flor. Esta escolha possibilitava uma estratégia de distribuição em diferentes espaços de circulação de mulheres (ônibus, escolas, shoppings) como um presente que lhe estava sendo dado.

Com base na referência de origami, prototipou-se uma flor, com o texto de chamada conceitual na parte externa (nas pétalas) e dentro quatro perguntas: (1) O que é o câncer de colo de útero?; (2) O que é o HPV?; (3) Como diagnosticar o câncer de colo de útero e (4) Como é o tratamento do câncer de colo de útero? Desdobrando cada uma das abas das perguntas encontram-se as respostas de cada uma destas questões em um texto direto e uma ilustração ao centro. A ilustração do centro mostrava uma personagem, a Rosa, contando a experiência que teve diante do câncer de colo de útero, partindo do autoexame, a relação com seus familiares, o diagnóstico e o sucesso do tratamento realizado. O protótipo foi inicialmente testado com aqueles do próprio grupo de pesquisa, que não haviam tido contato com o material gráfico até o momento. Neste teste, 
foi notado que a flor tinha um tamanho muito grande e que a história contida no centro da flor acabava por confundir a leitura das respostas, pois não ficava claro o que ler primeiro, se as respostas às perguntas ou a história da personagem. Não era possível entender se alguma sequência lógica entre os textos.

A partir deste primeiro teste, foram obtidos insights para solucionar os problemas constatados neste primeiro protótipo. A ideia pensada foi de, ao invés de dispor a história da personagem no centro da flor, que acabava por confundir a leitura, colocá-la atrás de uma das abas de pergunta, de forma que seu depoimento respondesse à pergunta. Para responder às demais três perguntas, foram desenhadas outras três personagens. A estratégia feita consistiu em tornar a leitura mais clara, lógica e, além disso, através dos depoimentos das mulheres, responder as dúvidas, tentando manter uma maior proximidade com o público, de forma que estas personagens respondem com linguagem coloquial e não tão impessoal como no primeiro protótipo, onde havia perguntas-respostas diretas. O resultado é reproduzido na Tabela 1.

Após pronto este segundo protótipo, foi realizado o primeiro teste junto mulheres na UBS e com outras da comunidade no entorno do Centro de Saúde. Neste momento foram levadas flores coloridas (com as pétalas rosas ou roxas ou brancas) e flores completamente em preto e branco. No primeiro momento as mulheres entrevistadas recebiam a flor em preto e branco, e nesta intervenção foi percebido que: As mulheres conseguiam perceber que 0 material representava uma flor; Elas demonstraram curiosidade ao ver a flor, porém parte delas hesitou em abri-la, não sabiam o que era para fazer ou tinham um pouco de receio em estragá-la; Quando foi solicitado que elas escolhessem uma cor para as pétalas da flor, a maior parte sugeria a cor rosa, pois representava mais a feminilidade e algumas se lembraram da campanha "Outubro Rosa", que utiliza a cor rosa para simbolizar mundialmente a luta contra o câncer de mama. Após as respostas, era mostrado a elas as flores coloridas, com pétalas rosas, roxas e brancas; Em relação aos textos, parte delas achou que as respostas eram extensas e como algumas estavam sem seus óculos, tiveram dificuldades para ler; Sobre o conceito da flor e a utilização de personagens femininas contando suas histórias, elas disseram terem gostado e que eram ideias criativas. 
Tabela 1.

Reprodução das histórias contidas no material educativo

\begin{tabular}{|c|c|}
\hline \multicolumn{2}{|c|}{ Histórias contidas no material educativo } \\
\hline $\begin{array}{l}\text { HISTÓRIA } 1 \text { - O que é o HPV? } \\
\text { Sou Tulipa tenho } 18 \text { anos, comecei } \\
\text { a namorar há algum tempo. Meu } \\
\text { parceiro usou camisinha algumas } \\
\text { vezes nas relações sexuais, e em } \\
\text { outras não. Durante o banho, } \\
\text { percebi que tinha uma verruga em } \\
\text { minha vagina. Me assustei e fui à } \\
\text { unidade básica de saúde, onde } \\
\text { descobri que esta verruga foi } \\
\text { causada pelo HPV, que é uma } \\
\text { doença sexualmente transmissivel. } \\
\text { o HPV também pode causar o } \\
\text { câncer de colo do útero. Realizei o } \\
\text { tratamento e não tenho mais a } \\
\text { verruga. Aprendi que é bom cuidar } \\
\text { da minha saúde sexual e me } \\
\text { prevenir de futuras doenças. }\end{array}$ & $\begin{array}{l}\text { HISTÓRIA } 2 \text { - Como faço para } \\
\text { diagnosticar o câncer de colo do } \\
\text { útero? } \\
\text { Sou Violeta tenho } 35 \text { anos, descuidei } \\
\text { da minha saúde, por me preocupar } \\
\text { com o meu trabalho, filhos e casa } \\
\text { para cuidar. Fiquei quatro anos sem } \\
\text { fazer o exame Papanicolau, não } \\
\text { sentia nada e achava que não tinha } \\
\text { necessidade. O Papanicolau, é um } \\
\text { exame rápido e simples, realizado } \\
\text { na Unidade Básica de Saúde. Este } \\
\text { exame, vai coletar material do fundo } \\
\text { da vagina, e este material é } \\
\text { analisado em laboratório.Com o } \\
\text { exame, descobri que tinha uma } \\
\text { lesão no útero. Realizei o tratamento } \\
\text { médico, a lesão desapareceu e não } \\
\text { se transformou em câncer. Levei um } \\
\text { susto, mas a partir de agora, não } \\
\text { vou descuidar da minha saúde. }\end{array}$ \\
\hline $\begin{array}{l}\text { HISTÓRIA } 3 \text { - O que é o câncer de } \\
\text { colo de útero? }\end{array}$ & $\begin{array}{l}\text { HISTÓRIA } 4 \text { - Qual é o tratamento } \\
\text { do câncer de colo de útero? }\end{array}$ \\
\hline $\begin{array}{l}\text { Sou Margarida, tenho } 66 \text { anos. } \\
\text { Sempre cuidei da minha saúde. } \\
\text { Como nunca tive nenhuma alteração } \\
\text { no exame de Papanicolaou, não } \\
\text { preciso mais realiza-lo. Tenho duas } \\
\text { netas, de } 12 \text { e } 13 \text { anos, e descobri } \\
\text { que elas podem receber a vacina } \\
\text { contra o HPV, na unidade de saúde. } \\
\text { o HPV pode causar uma lesão no } \\
\text { útero. Essa lesão, se não for tratada, } \\
\text { pode se transformar em câncer. Que } \\
\text { é um tipo de câncer que se } \\
\text { desenvolve no colo do útero. No } \\
\text { início, a mulher não sente nada. } \\
\text { Porém, se a lesão aumentar, a } \\
\text { mulher pode ter sangramentos, } \\
\text { corrimento e dor pélvica. Aprendi } \\
\text { que da minha saúde cuido eu. }\end{array}$ & $\begin{array}{l}\text { Olá, eu sou Rosa, tenho } 48 \text { anos. } \\
\text { Sempre tive medo de fazer o exame } \\
\text { Papanicolau, porque diziam que } \\
\text { doía. Ninguém da minha familia } \\
\text { tinha tido câncer, por isso nunca me } \\
\text { preocupei. Há alguns anos, minha } \\
\text { filha me convenceu a fazer o exame. } \\
\text { Confesso que senti um pouco de } \\
\text { desconforto, mas valeu a pena. Foi } \\
\text { quando descobri que tinha Câncer } \\
\text { do Colo do útero. Como descobri } \\
\text { cedo, fiz o tratamento adequado. O } \\
\text { tratamento depende muito do } \\
\text { tamanho da lesão no útero, os mais } \\
\text { comuns são cirúrgico e/ou } \\
\text { radioterapia. Hoje estou curada e } \\
\text { muito bem. Aprendi que da minha } \\
\text { saúde cuido eu. }\end{array}$ \\
\hline
\end{tabular}

\section{DISCUSSÃO}

O material educativo, entendido como uma tecnologia social leve-dura ( $\mathrm{Da}$ Ros et al., 2012), foi desenvolvido a partir da necessidade identificada pelos pesquisadores de auxiliar as mulheres no processo de conhecimento do próprio corpo, manutenção da sua saúde e prevenção do CCU. Este permite dar significado aos dilemas, dúvidas e questionamentos das usuárias, favorecendo o compartilhamento de experiências e resolução de problemas (Marques, Tyrrell, \& Oliveira, 2013).

O processo de trabalho das equipes de atenção primária tem como um dos seus focos o desenvolvimento de ações educativas que possam intervir no processo de saúde-doença. Os materiais impressos, assim como as companhas 
de mídia, não visam substituir a orientação do profissional, mas atrair a população aos serviços de prevenção de doenças. O conteúdo que é transmitido somente é aplicado no cotidiano das pessoas, quando o mesmo é assunto de debate, troca de saberes e experiências (Fundação Nacional de Saúde, 2007). Sendo assim, o material educativo visa auxiliar no processo de construção do saber, como meio de chamar a atenção para o assunto, levantando questionamentos e preocupações a respeito da saúde.

Os dilemas criados para o material educativo colocam as usuárias em situações do cotidiano que, além de prestar informações sobre o CCU, trabalham a percepção de risco de vir a ter a doença (Leventhal et al., 1997). As histórias apresentadas têm potencial para desestabilizar as crenças relacionadas ao CCU nas usuárias, favorecendo que elas percebam potenciais riscos para sua saúde que antes poderiam ser negligenciados, ativando o processo autorregulatório. 0 ajuste da autorregulação proporcionado poderá contribuir para que as mulheres empreendam condutas preventivas sistemáticas e autossustentáveis com relação ao CCU. Naturalmente, a maior ou menor ativação deste sistema de autorregulação dependerá também do nível de conhecimento sobre a doença, de experiências pregressas diretas ou indiretas e de aspectos relacionados ao sistema de saúde (por exemplo: relação profissional-paciente, acesso ao serviço de saúde e aos exames preventivos, etc.).

No que tange à pesquisa realizada pela equipe de design no contexto natural (unidade de saúde), pôde-se perceber que a maior parte dos materiais gráficos manipulados pelas mulheres eram cartilhas ou cartazes que traziam bastante conteúdo. Em alguns casos, devido a grande quantidade textual e da forma de publicação, os materiais não tinham boa legibilidade, o que dificultava a compreensão por parte do público. Outra característica em comum dos materiais em questão era a utilização de imagens que não eram atrativas, impactantes ou diretamente relacionadas ao que se estava comunicando. No entanto, a funcionalidade dos materiais é alcançada pela combinação de linguagens (visual e verbal) a fim de que sejam complementares na busca pela otimização e eficácia do processo de entendimento (Roam, 2008). Portanto, é necessário que haja relações diretas nestes materiais, caso contrário, forma-se um hiato entre o que se quer comunicar e a forma como são mediadas as informações. As imagens presentes "devem ser usadas como propósito estratégico, não apenas para dividir o texto ou deixar a página menos sem graça; não são elementos subordinados os textos, mas uma parceria - entre o visual e o verbal" (White, 2006).

A maior valorização e empregabilidade deste tipo de material pode ser realizada no momento em que a sua construção considere aspectos subjetivos, culturais, sociais do público alvo. As escolhas projetuais que formalizam o conteúdo no material impresso consideraram esses aspectos para que o público selecionasse o material para ler. Quando o público se identifica com o material 
produzido, ele pode auxiliá-lo na tomada de decisões em saúde (Santos, 2009). Nesse sentido, o material educativo aqui proposto possui embasamento teórico sólido, pois o modelo de autorregulação em saúde é amplamente aceito e difundido no contexto científico (Figueiras et al., 2010; Castro, Aretz, Lawrens, Romeiro, \& Haas, 2013; Green, Steinnagel, Morris, \& Laakso, 2010). Existe um corpo de evidências bem constituído sobre esse modelo, tanto com populações clínicas e de pessoas sadias, que subsidiam a compreensão profunda do fenômeno - a prevenção e o autocuidado das mulheres.

Nenhuma referência que descrevesse a elaboração de materiais educativos na área da saúde agregando conhecimento de diferentes especialidades foi identificado. Os estudos que descrevem a construção de materiais são realizados geralmente pela área da enfermagem (Cariolano-Marinus, Pavan, \& Bettencourt, 2014; Gonçalves, Barbieri, \& Gabrielloni, 2008; Reberte, Hoga, \& Gomes, 2012).

\section{CONSIDERAÇÕES FINAIS}

A proposta deste trabalho foi de elaborar um material educativo que proporcionasse identificação com as mulheres usuárias de UBS e pudesse ampliar o conhecimento da mulher sobre a possibilidade de autocuidado frente ao CCU. Na elaboração do material, buscou-se utilizar uma forma diferente de comunicação com as usuárias, em que elas se identificassem com a problemática e com as características de pelo menos uma das personagens, o que torna a problemática mais próxima da sua vida cotidiana. A metodologia do humancentred design (centrada no ser humano) que busca traduzir a realidade das pessoas nos materiais desenvolvidos e testá-los, mostrou-se útil na criação das vinhetas que tinham essa característica de aproximar o material educativo das dúvidas e problemáticas da saúde das mulheres.

Ressalta-se que o material educativo criado é inovador porque foi construído a partir de resultados de pesquisa empírica com a população alvo e utilizou conhecimentos do design na sua elaboração, unindo saberes e aproximando áreas. A participação ativa das mulheres e dos profissionais da saúde na construção do material é outro ponto a ser destacado e que mostra a importância e aplicabilidade do material.

Como limitação do estudo, percebe-se que o material não é acessível a toda a população, como mulheres analfabetas e analfabetas funcionais. Além disso, o fato de a elaboração do material ter tido como base somente uma UBS talvez tenha excluído particularidades de centros de saúde de outras regiões. Apesar dessas questões, destaca-se que o material educativo foi bem avaliado pelas mulheres e profissionais da saúde, podendo ser um material auxiliar na busca pela prevenção do câncer de colo de útero. 


\section{REFERÊNCIAS}

Bish, A., Ramirez, A., Burgess C., \& Hunter, M. (2005). Understandig why women delay in seeking help for breast cancer symptoms. Journal of Psychos Research, 58(4), 321-326. doi: 10.1016/j.jpsychores.2004.10.007

Brito-Silva, K., Bezerra, A.F.B., Chaves, L.D.P., \& Tanaka O.Y. (2013). Integralidade no cuidado ao câncer do colo do útero: avaliação do acesso. Revista de Saúde Pública, 48(2), 240-248. Recuperado de: http://www.revistas.usp.br/rsp/article/view/81147/84771.

Castro, E.K., Aretz, M., Lawrens, P., Romeiro, F.B., \& Haas, S.A. (2013). Illness perceptions in brasilian women whit a cervical cancer, women with a precursory lesions and healthy women. Psicooncología, 10(2-3), 417-423. doi: $10.1590 / 1678-7153.20$

Corazza, C. (2014). Psicoeducação para a prevenção do câncer de colo de útero: Uma Proposta de Intervenção. Dissertação de Mestrado, Universidade do Vale do Rio dos Sinos, São Leopoldo.

Coriolano-Marinus, M.W.L., Pavan, M.I., \& Bettencourt A.R.C. (2014). Validação de material educativo para alta hospitalar de pacientes com prescrição de oxigenoterapia domiciliar prolongada. Escola Anna Nery, 18(2), 284-289. doi: $10.5935 / 1414-8145.20140041$

Cruz, L., \& Loureiro, R. (2008). A comunicação na abordagem preventiva do câncer do colo do útero: importância das influências histórico-culturais e da sexualidade feminina na adesão às campanhas. Saúde \& Sociedade, 17(2), 120-131. doi: 10.1590/S0104-12902008000200012

Da Ros, M.A., Maeyama, M.A., \& Leopardi M.T. (2012). Tecnologia na área da saúde. De que tecnologia estamos falando? Saúde \& Transformação Social, 3(3), 29-35. Recuperado de http://www.redalyc.org/pdf/2653/265323673006.pdf

Figueiras, M., Marcelino, D.S., Claudino, A., Cortes, M.A., Maroco, J., \& Weinman, J. (2010). Patients' illness schemata of hypertension: The role of beliefs for the choice of treatment. Psychology \& Health, 25(4), 1-11. doi: 10.1080/08870440802578961.

Freitas, R., Waechter, H., Coutinho, S., \& Gubert, F. (2014). Design da Informação, Comunicação, Saúde e prevenção das DST/Aids: estudo sistemático. Comunicação e Sociedade, 35(2), 291-314. doi: 10.15603/2175-7755/cs.v35n2p291-314

Fundação Nacional de Saúde. (2007). Diretrizes de educação em saúde visando à promoção da saúde: documento base ( $2^{\mathrm{a}}$ ed.). Brasília, DF: FUNASA.

Gonçalves, M., Barbieri, M., \& Gabrielloni, M. (2008). Teste de Papanicolaou: construção e validação de material educativo para usuárias de um serviço de saúde. Saúde Coletiva, 20(5), 39-44. Recuperado de: http://www.redalyc.org/pdf/842/84202003.pdf 
Green, H.J., Steinnagel, G., Morris, C., \& Laakso, E.L. (2014). Health behaviour models and patient preferences regarding nutrition and physical activity after breast or prostate cancer diagnosis. European Journal Cancer Care, 23(5), 640-652. doi: 10.1111/ecc. 12190.

IDEO. (2009). The Human Centred Design Toolkit. Londres: IDEO.

INCA. (2016). Câncer Cervical. Rio de Janeiro. Rio de Janeiro: INCA.

Leventhal, H., Benyamini, Y., Brownlee, S., Diefenbach, M., Leventhal, E., \& Patrick-Miller, L. (1997). Illness Representations: Theoretical Foundations. In Petrie K., \& Weinman, J. Perceptions of Health \& IIlness (pp. 19-45). London: Harwood Publishers.

Mantenese, J.C. (2008). Câncer Ginecológico: ovário, útero e vagina. In Carvalho, V.A., Franco, M.H., Kovács M.J., Liberato R.P., Macieira R.C., Veit M.T. ..., \& Barros L.H. Temas em Psico-Oncologia (pp. 59-66). São Paulo: Summus editorial.

Marques, S.C., Tyrrell, M.A., \& Oliveira, D.C. (2013). As práticas educativas na prevenção do HIV/AIDS das usuárias da rede básica de saúde do Rio de Janeiro/Brasil. REME, 17(3), 538-546. doi: 10.5935/1415-2762.20130040

Marteau T., \& Weinman J. (2013). Self-regulation and the behavioural response to DNA risk information: a theoretical analysis and framework for current research and future practice. Social Science \& Medicine, 62(6), 1360-1368. doi: 10.1016/j.socscimed.2005.08.005

Ministério da Saúde. (2012). Política Nacional de Atenção Básica (1a ed.). Brasília, DF: DAB.

Ministério da Saúde. (2013). Controle dos cânceres do colo do útero e da mama (2a ed.). Brasília, DF: DAB.

Ministério da Saúde. (2015). Começa oferta da vacina contra HPV para meninas de 9 a 11 anos. Recuperado de: http://www.brasil.gov.br/saude/2015/03/comeca-oferta-da-vacina-contrahpv-para-meninas-de-9-a-11-anos

OMS. (2013). Prevenção e controle de amplo alcance do câncer do colo do útero: um futuro mais saudável para meninas e mulheres ( $1^{a}$ ed.). Washington, DC: OPAS.

Reberte, L., Hoga, L., \& Gomes, A. (2012). O processo de construção de material educativo para a promoção da saúde da gestante. Revista Latino Americana de Enfermagem, 20(1), 101-108. Recuperado de http://www.scielo.br/pdf/rlae/v20n1/pt_14.pdf

Rees, G., Fru, A., Cull, A., \& Sutton S. (2004). Illness perceptions and distress in women at increased risk of breast cancer. Psychology \& Health, 19(3), 749765. Recuperado de http://www.phpc.cam.ac.uk/pcu/wpcontent/files/2012/01/Paper94.pdf 
Pimenta, D.N. (2008). Disseminação de informação sobre dengue: o ergodesign no desenvolvimento e avaliação de material multimídia para educação em saúde. Tese de Doutorado, Fundação Oswaldo Cruz, Belo Horizonte.

Roam, D. (2008). The back of the napkin: solving problems and selling ideas with pictures. New York: Penguin Group.

Santos, A.K., Monteiro, S., \& Rozember B. (2009). Significados e usos de materiais educativos sobre hanseníase segundo profissionais de saúde pública do Município do Rio de Janeiro, Brasil. Caderno de Saúde Pública, 24(4), 857-867. doi: 10.1590/S0102-311X2009000400017

Shiloh, S. (2006). Illness Representations, Self-Regulation, and Genetic Couseling: A Theoretical Review. Journal of Genetic Counseling, 15(5), 325337. doi: 10.1007/s10897-006-9044-5

Shiloh S., Drori E., Orr-Urtreger A., \& Friedman E. (2009). Being 'at-risk' for developing cancer: cognitive representations and psychological outcomes. Journal of Behavioral Medicine, 32(2), 197-208. doi: 10.1007/s10865-0089178-z

Tavafian, S.S. (2012). Predictors of Cervical Cancer Screening: An Application of Health Belief Model. Recuperado de: http://www.intechopen.com/books/topics-on-cervical-cancer-with-anadvocacy-for-prevention

White, J. (2006). Edição de design: para designers, diretores de arte e editores o guia clássico para ganhar leitores. São Paulo: JSN EDITORIAL LTDA; 2006. 
Sobre os autores

Ana Carolina Peuker é psicóloga, tem Mestrado, Doutorado e Pós Doutorado em Psicologia pela Universidade Federal do Rio Grande do Sul (UFRGS). É professora Associada do Programa de Pós-Graduação em Psicologia da UNISINOS, pesquisadora de Pós doutorado do GEAPSA (Grupo de Estudos Avançados em Psicologia da Saúde). acpeuker@hotmail.com

Natália Britz de Lima é enfermeira pela Universidade do Vale do Rio dos Sinos. No momento é Residente de Enfermagem no Grupo Hospitalar Conceição. nataliabritz@gmail.com

Karine de Mello Freire é graduada em Administração, com mestrado em Administração (UFRGS) e Doutorado em Design (PUCRJ), com estágio doutoral sanduíche na Lancaster University. É professora do Programa de Pós-Graduação em Design da UNISINOS. kmfreire@unisinos.br

Caio Marcelo Miolo de Oliveira é graduado em Desenho Industrial e é mestrando em Design Estratégico na UNISINOS. caiomarcelo.mo@email.com Elisa Kern de Castro é psicóloga, Mestre em Psicologia (UFRGS), Doutora em Psicologia Clínica e da Saúde pela Universidade Autônoma de Madrid e estágio pós-doutoral em Psicologia pela Universidade de Salamanca. Coordenadora do GEAPSA, Professora do Programa de Pós-graduação em Psicologia da UNISINOS, pesquisadora do CNPq. elisakc@unisinos.br

Recebido em: 30/03/2016

Revisado em: 23/06/2016

Aceito em: 18/09/2016 